\title{
Mind wandering during film comprehension: The role of prior knowledge and situational interest
}

\author{
Kristopher Kopp $^{1} \cdot$ Caitlin Mills $^{1} \cdot$ Sidney D'Mello ${ }^{1}$
}

Published online: 28 September 2015

(C) Psychonomic Society, Inc. 2015

\begin{abstract}
This study assessed the occurrence and factors that influence mind wandering (MW) in the domain of film comprehension. The cascading model of inattention assumes that a stronger mental representation (i.e., a situation model) during comprehension results in less MW. Accordingly, a suppression hypothesis suggests that MW would decrease as a function of having the knowledge of the plot of a film prior to viewing, because the prior-knowledge would help to strengthen the situation model during comprehension. Furthermore, an interestmoderation hypothesis would predict that the suppression effect of prior-knowledge would only emerge when there was interest in viewing the film. In the current experiment, 108 participants either read a short story that depicted the plot (i.e., priorknowledge condition) or read an unrelated story of equal length (control condition) prior to viewing the short film (32.5 minutes) entitled The Red Balloon. Participants self-reported their interest in viewing the film immediately before the film was presented. MW was tracked using a self-report method targeting instances of MW with metacognitive awareness. Participants in the priorknowledge condition reported less MW compared with the control condition, thereby supporting the suppression hypothesis. MW also decreased over the duration of the film, but only for those with prior-knowledge of the film. Finally, prior-knowledge effects on MW were only observed when interest was average or high, but not when interest was low.
\end{abstract}

Keywords Mind wandering $\cdot$ Attention $\cdot$ Film comprehension $\cdot$ Situational interest

\section{Kristopher Kopp}

kkopp@nd.edu

1 Department of Psychology, University of Notre Dame, 203 Haggar Hall, Notre Dame, IN 46556, USA
Mind wandering (MW) is defined as an attentional shift away from a primary task or ongoing event in the external environment towards internally generated task-unrelated thoughts or feelings (McVay \& Kane, 2010; 2012; Smallwood \& Schooler, 2006; 2015). MW has been studied in a variety of contexts including standard lab tasks, reading, and during everyday routine activities (Smallwood \& Schooler, 2015). Tasks that require low levels of engagement have resulted in more MW than tasks that are highly engaging (Smallwood, Fishman, \& Schooler, 2007). In particular, MW occurs $40 \%$ to $50 \%$ of the time during minimally engaging lab tasks, such as signal detection tasks (Smallwood et al., 2007), and while viewing online lectures (Risko, Buchanan, Medimorec, \& Kingstone, 2013). It is less frequent (approximately 30\%) in tasks that require a comparatively deeper level of engagement, such as reading (Smallwood et al., 2007). An interesting question pertains to the incidence of MW in contexts that are intentionally designed to sustain attention, such as viewing films. Film makers of commercially produced films apply cinematic devices for the purpose of keeping the viewer engaged (Bordwell, 2013). Film viewing also involves processing information from multiple modalities (i.e., audio and visual), which would presumably aid in keeping attention focused. Furthermore, films are a universally enjoyed cultural and educational medium. Although previous research has investigated content of thoughts and MW while viewing films (Hurlburt, Lech, \& Saltman, 1984; Reise \& Hurlburt, 1987), MW rates were not reported so little is known regarding the extent to which MW occurs during film comprehension. For these reasons, we considered film comprehension to be a worthy context to study MW.

Much of what we know regarding MW during comprehension comes from research on reading. There is evidence, however, that film and reading comprehension share similar cognitive processes (Magliano, Loschky, 
Clinton, \& Larson, 2013; Magliano, Miller, \& Zwaan, 2001) so a degree of extrapolation from reading to film is appropriate. Comprehension, in general, involves hierarchical cognitive process where information is encoded at a shallow level and proceeds through the cognitive system resulting in a mental representation or a situation model (Zwaan \& Radvansky, 1998). At the shallow levels, information is encoded and then transformed into a deeper "propositional" or "gist" like representation. This information is then incorporated with prior or existing knowledge at the situation model level, which is thought to be a crucial step for deep understanding (Graesser, Singer, \& Trabasso, 1994). The relationship between the situation model and comprehension is dynamic in that the situation model is constantly being updated and the model directs attention in a top-down fashion to provide a context in which events are interpreted (McNamara \& Magliano, 2009).

It is assumed that there is a negative relationship between the strength of the situation model and frequency of MW. Specifically, the cascading model of inattention (Smallwood, 2011; Smallwood et al., 2007) assumes that stronger situation models reduce the propensity to MW. According to this perspective, MW can have a detrimental influence on the creation of the situation model during comprehension, because MW impairs the ability to encode information at the shallow levels (Smallwood, 2011; Smallwood et al., 2007). The effect of a lack of encoding information at the shallow levels "cascades" through the cognitive system hindering the creation of the situation model. Thus, having a superior situation model would reduce the likelihood of the cascading effect during comprehension and potentially help to sustain attentional focus by directing attention in a top-down fashion and reduce MW.

In line with the cascading model, we were interested in assessing the extent to which having prior knowledge regarding the plot of a film would suppress MW. Based on the above discussion, if a superior situation model results in fewer instances of MW, then we would expect that having the knowledge of the plot of the film (i.e., prior knowledge) would result in a superior situation model. This would presumably reduce the propensity for cascading effects of inattention and therefore suppress MW because the superior situation model would increase attentional focus. We refer to this hypothesis as the suppression hypothesis.

In addition to prior-knowledge, interest is an important factor to consider in the context of film comprehension since film viewing often is guided by interest. MW has been shown to negatively correlate with interest (Giambra \& Grodsky, 1989; Smallwood, Nind, \& O'Connor, 2009). Our focus is on situational interest which is based on immediate contextual or environmental information rather than other forms of interest (Hidi, 2001; Linnenbrink-Garcia et al., 2010). In the present context, situational interest would be the extent to which the individual is interested in viewing a specific film. We hypothesize that situational interest would moderate the effects of prior-knowledge on MW (interest-moderation hypothesis). In particular, it might be the case that having priorknowledge could decrease MW, but only when interest is sufficiently high. In the absence of interest, prior-knowledge may not matter at all because the lack of interest would place the focus of attention elsewhere.

We tested these hypotheses by conducting an experiment where participants self-reported MW while watching the film "Le Ballon Rouge" (English translation: The Red Balloon). This is a short film (released in 1956) with a musical score and very little dialogue. Before the presentation of the film, the prior-knowledge condition was presented with a text version of the film which served as supplying the plot of the film. A control condition was presented with a story unrelated to the film. We hypothesize that MW should be lower in the priorknowledge condition (suppression hypothesis), but only when interest is sufficiently high (interest moderation hypothesis).

\section{Method}

\section{Participants}

A total of 108 college students from a private Midwestern university $(n=65)$ and a public university in the southern United States $(n=43)$ participated for partial course credit ( $66 \%$ female; average age $=20.1$ years). The sample size was selected in an attempt to detect a moderate effect of condition $(f=.30)$ with a statistical power of 0.80 and a two-tailed alpha of 0.05 , using $\mathrm{G}^{*}$ Power (Faul, Erdfelder, Lang, \& Buchner, 2007) for the requisite computation.

\section{Materials}

\section{Film}

Participants viewed the narrative film "The Red Balloon"- a 32.5-minute French film with English subtitles. The film takes place in Paris and conveys the story of a young boy who finds a red helium-filled balloon that follows him wherever he goes. We selected this film, because it is an older film and the likelihood that participants would have been exposed to the film is low. It also has been used in film comprehension studies (Zacks, Speer, Swallow, \& Maley, 2010).

\section{Stories}

The between-subjects experimental manipulation consisted of participants reading one of two stories before watching the "The Red Balloon." Participants in the prior-knowledge condition read the text version of "The Red Balloon" by Albert 
Lamorisse (1978). The story conveyed the same major plot lines as the film. Participants in the control condition read "Bernie the Early Bloomer" by Karyn Smith (1999). This story was about three boys attempting to make a youth baseball team and was not related to "The Red Balloon" film. Both stories were approximately equal in length (Red Balloon $=1$, 774 words; Early Bloomer = 1,892 words). "Bernie the Early Bloomer" was modified so that both stories were equal in terms of text complexity as assessed by the Flesh Kincaid Grade Level (FKGL) metric (Klare, 1974), (FKGL for The Red Balloon $=4.4$; FKGL for Early Bloomer $=4.4$ ).

\section{Interest assessment}

Participants were asked to self-report their interest in viewing the film by completing a three question interest inventory before viewing the film. The creation of this inventory was based on constructs related to situational interest (i.e., Linnenbrink Garcia et al., 2010). Specifically, participants were asked about their interest in viewing the film, curiosity about the film, and whether or not they thought that the film would hold their attention (scales ranging from 1 [low] to 6 [high]).

\section{Comprehension assessment}

Participants completed a comprehension assessment after viewing the film. The assessment consisted of 13 multiple choice items with 4 options (the correct answer and 3 foils). The items were experimenter generated and were designed to assess comprehension of the events that occurred in the film and focused on surface-level facts rather than deep inferences.

\section{Design and procedure}

All instructions and experimental materials were administered via computer. Participants were randomly assigned to the prior knowledge or control condition and read the respective story for an average of 7 minutes using a self-paced reading paradigm. After reading, all participants were informed that they would be viewing the film "The Red Balloon" and asked to report their interest in viewing the film. Participants were asked to self-report MW throughout viewing the film. A small beep sounded to register their response but film play was not interrupted. We chose to have participants self-report MW instead of the more traditional probing method (Smallwood $\&$ Schooler, 2006), because we were interested in the individual's metacognitive awareness of MW (Schooler, Reichle, \& Halpern, 2004). Furthermore, the probe method has the potential to interrupt the comprehension process (i.e., when participants report "no" to the probes), which is particularly problematic as participants did not have control over the film presentation (i.e., no pausing or rewinding capabilities were available). We considered the distinction between two types of MW, because previous research (Smallwood, Obonsawin, \& Heim, 2003; Stawarczyk et al. 2011) suggests that disruptions during comprehension also could occur due to appraisals of the task (i.e., not necessarily a task unrelated thought, but not paying attention to the content either) (Smallwood, Obonsawin, \& Heim, 2003). Thus, we considered MW as stimulus independent thoughts that are decoupled from the stimuli of the current task and task-unrelated in that they are unrelated to the current activity (i.e., TUTs). We also considered stimulus independent and task-related interferences (i.e., TRIs), such as an appraisal of the task. Participants were asked to report a TUT if they were "thinking about anything else besides the movie." Participants were explicitly instructed to report a TRI while if they were "thinking about the task itself but not the actual content of the movie." Participants were instructed to report MW by pressing specific labeled keys on the keyboard (i.e., one key for TUT and a separate key for TRI).

\section{Results}

A two-tailed alpha of 0.05 was adopted for all significance testing. We considered the possibility that there may be differences between the two sampled populations on variables that were not directly measured. To alleviate these concerns, all analyses reported below were conducted after z-score standardizing each variable by school (see Table 1 for unstandardized data). Furthermore, there were no significant differences among conditions regarding any of the demographic variables: age $(p>$ $0.05)$; gender $(p>0.05)$. As mentioned above, we considered the distinction between two types of MW (i.e., TUTs and TRIs). We found no significant effect of MW type, and there was not a significant interaction between type and condition. Thus, all analyses are conducted with overall MW reports.

\section{Overall MW}

We first assessed the extent to which MW occurred during the film. All participants viewed the film for 32.5 minutes and reported MW an average of 12.43 times $(S D=15.61)$ with a range of 0 to 71 . This is equivalent to 0.38 times per minute $(S D=0.47)$ or once every 2.6 minutes.

Table 1 Mean MW (raw totals) and comprehension performance scores (proportion) by condition (with standard deviations in parentheses)

\begin{tabular}{llll}
\hline $\begin{array}{l}\text { Dependent } \\
\text { variable }\end{array}$ & $\begin{array}{l}\text { Prior-knowledge } \\
\text { condition }\end{array}$ & $\begin{array}{l}\text { Control } \\
\text { condition }\end{array}$ & Overall MW \\
\hline MW total & $10.05(13.76)$ & $14.98(16.67)$ & $12.43(15.36)$ \\
$\begin{array}{l}\text { Comprehension } \\
\text { assessment }\end{array}$ & $0.93(.117)$ & $0.90(.132)$ & $0.91(.125)$ \\
\hline
\end{tabular}




\section{Effect of prior-knowledge on mind wandering}

One-way ANOVA with condition as a between subject factor revealed that the prior-knowledge condition resulted in significantly less MW than the control condition, $F(1,106)=$ 4.18, $p=0.04, M S E=0.961, d=0.39, \eta^{2}=0.05$. We then explored and assessed the patterns of MW over the duration of the film. We first computed whether MW occurred at least once in each minute of the film and then analyzed how MW occurrence varied as a function of duration in each condition (see Appendix A for proportion of MW reports for each minute by condition). Next, we computed a mixed-effect item-level (or minute-level in this case) logistic regression model (Pinheiro \& Bates, 2000) using the lme4 package in R (Bates \& Maechler, 2010). The dependent variable was MW occurrence in each minute (coded as 0 for no vs. 1 for yes). The intercept-only random effect was participant. The interaction term of school affiliation (categorical factor with two levels) $\times$ condition (categorical factor with two levels) $\times$ time (continuous variable ranging from 0 to 33 minutes in 1minute increments) was added to assess if the effect of duration on MW varied as a function of condition. School was included in the interaction term to assess if patterns varied by population.

The three-way interaction between school, condition, and time was not significant, $p>0.05$, suggesting that the trends of each condition were similar for both populations. The interaction between condition and duration was significant, $F(1,3556)=6.65, p=0.01$ (Fig. 1). Posthoc analyses indicat-

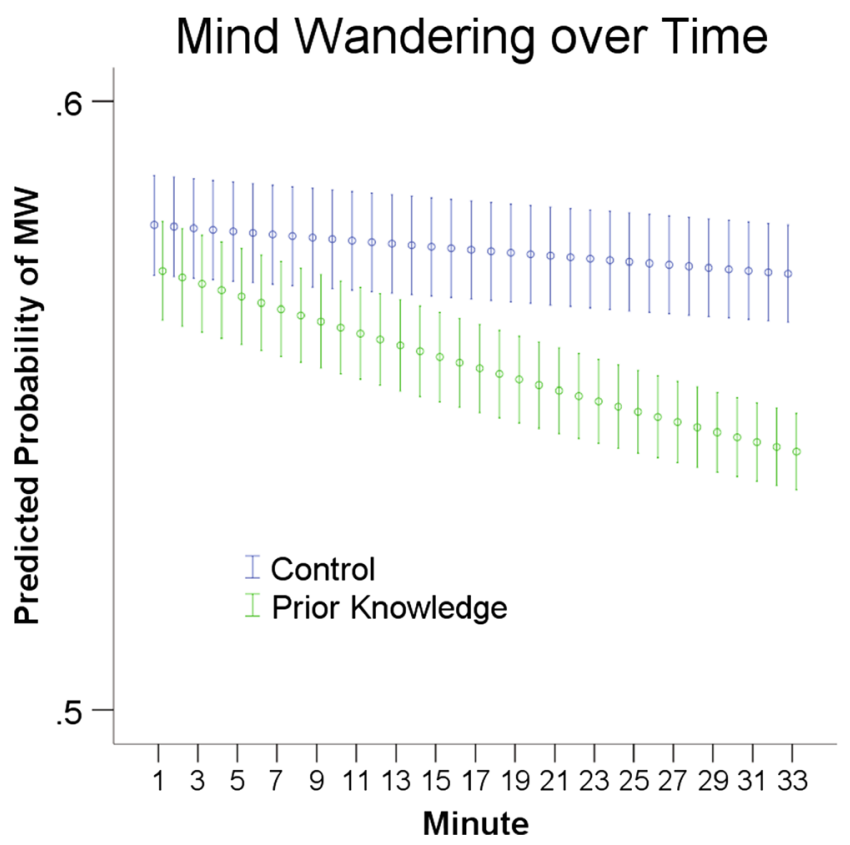

Fig. 1 Plot showing mean predicted probability of MW over time for each condition. Error bars represent 1 standard error ed that duration was not a significant predictor of $\mathrm{MW}$ for the control condition, $F(1,1712)=1.08, p=0.30, B=-0.013$, $S E=0.011$ but was a significant negative predictor of $\mathrm{MW}$ for the prior-knowledge condition, $F(1,1844)=19.16, p=0.009$, $B=-0.03, S E=0.012$, suggesting that MW decreased with time when the plot was known. Thus, having knowledge of the plot of the film before viewing suppressed MW throughout the duration of the film.

\section{Situational interest and mind wandering}

The three questions of the situational interest measure demonstrated sufficient reliability (Cronbach's alpha $=0.88$ ). The scores were averaged to construct one previewing situational interest measure and then standardized by school. The priorknowledge condition and the control condition did not significantly differ on ratings of interest, $p>0.05$. The PROCESS macro for SPSS (Preacher \& Hayes, 2004) was used to assess the moderating effect of situational interest on the relationship between condition and MW. We found that interest did have a moderating effect $(B=-0.44,95 \% \mathrm{CI}[-0.8544$ to -0.0285$]$, $t=2.12, p=0.03)$. Accordingly, a simple slopes analysis (Fig. 2) indicates that when interest was high (i.e., one standard deviation above the mean) the prior-knowledge condition reported significantly less $\mathrm{MW}$ than the control condition $(B=-0.82,95 \%$ CI $[-1.4306$ to -0.2233$], p<0.01)$. This same pattern occurred when interest was at the mean $(B=$ $-0.46,95 \%$ CI $[-0.8807$ to -0.0358$], p=0.03)$. However, there was no difference between conditions when interest was low (i.e., one standard deviation below the mean), $(B=-0.09$, 95\% CI $[-0.5697$ to 0.3906$], p=0.71)$. Thus, having prior-knowledge resulted in less MW during film comprehension, but only for those who have interest in viewing the film, thereby supporting the interest moderation hypothesis.

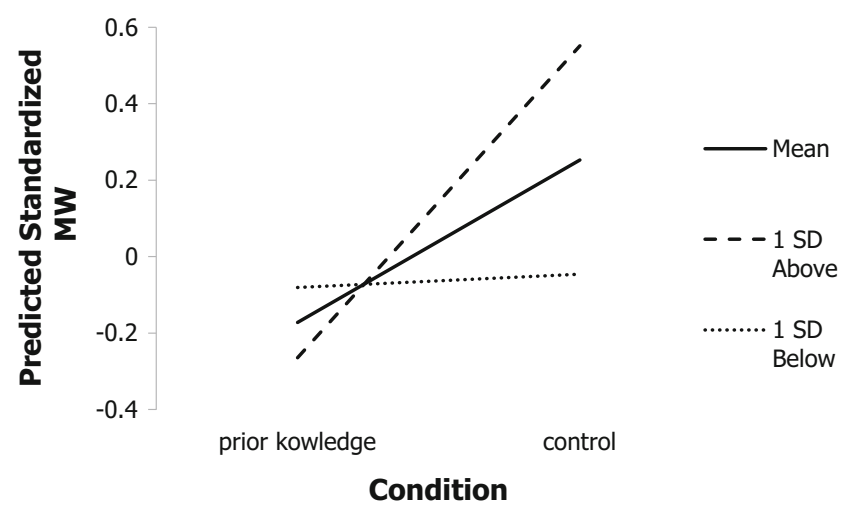

Fig. 2 Interaction plot of predicted standardized MW reports at low, average, and high interest by condition 


\section{Comprehension}

We next considered comprehension of the film. The comprehension assessment was scored by assessing the proportion of answers correctly answered. The correlation between $\mathrm{MW}$ and comprehension performance was negligible and nonsignificant $(r=-0.024, p=0.81)$. The unstandardized scores (Table 1) revealed that there was a ceiling effect. There were no significant differences of standardized scores between the priorknowledge condition $(M z=0.18, S D=0.67)$ and the control condition $(M z=0.01, S D=0.71), F(1,105)=$ $1.73, p=0.19, M S E=0.481, d=0.11$, although the trend was in favor of the prior-knowledge condition.

\section{Discussion}

We assessed MW in the context of film comprehension with the goals of (1) assessing the extent to which MW occurs, (2) assessing the impact of prior-knowledge on MW, and (3) assessing the influence of situational interest on the relationship between prior-knowledge and MW. In the remainder of this section, we take stock of the major findings, consider their theoretical implications, and discuss limitations and avenues of future work.

Our first finding was that MW occurred roughly once every 2.5 minutes while viewing the 32.5 -minute film. Previous research has shown that MW differs across contexts that vary with regard to the amount of engagement required of the individual. For example, there is a high propensity to MW during minimally engaging tasks (i.e., signal detection tasks) that require superficial levels of attention (i.e., Smallwood et al., 2007). We assumed that film comprehension would be engaging and require a moderate amount of attention, and the results seem to confirm this assumption. However, it is difficult to make concrete comparisons across studies, because we did not compare MW rates across tasks. Future research would benefit from comparing MW during film comprehension to other contexts.

The cascading model of inattention (Smallwood, 2011; Smallwood et al., 2007) assumes that a stronger situation model results in less MW. Accordingly, we tested a suppression hypothesis that posits that priorknowledge would have a suppressing effect on MW. We found support for this hypothesis in that knowing the plot of the film resulted in fewer instances of selfcaught MW compared with a control condition that did not have knowledge of the plot. A follow-up analysis revealed that the propensity to MW decreased over the duration of the film when the plot was known. This observed effect suggests that the superior situation model may have suppressed MW over time. The inferior situation model fails to direct attention in a top-down fashion resulting in a higher propensity for attention to drift and MW to occur.

We also investigated how situational interest would impact the relationship between prior-knowledge and MW by testing an interest moderation hypothesis. We found that priorknowledge reduced MW but only for those who were interested in viewing the film. Providing prior-knowledge alone did not reduce MW, because there were no differences between conditions when interest was low. It is likely that for some individuals, the Red Balloon story was simply not interesting.

Like any study, this research is not without limitations. First, we used a short film in relation to many feature length films. A longer film may reduce any effect of prior-knowledge, so replication with longer films is warranted. Second, "The Red Balloon" has very little dialogue. The interpretation of the plot is almost purely based on visual perception. It is rare to find a modern commercial film with little to no dialogue. Future research may benefit from assessing MW using this experimental design with a film with more dialogue. Third, we found no relationship between MW and comprehension and no effect of condition on comprehension. This finding does not align with previous research which has consistently shown a negative relationship between MW and comprehension (Smallwood et al., 2009; Smallwood \& Schooler, 2015). One possible reason for this finding is that our assessment was too easy, thereby resulting in the ceiling effect, and no meaningful variability. It may be beneficial for future research to investigate the relationship between prior-knowledge and MW and film within the context of film using an assessment that taps a deeper level of knowledge.

In conclusion, we have shown that even though film viewing may be a very engaging context for maintaining attention, MW does indeed occur. Our results also suggest that it is possible to decrease MW during film comprehension by simply providing the viewer with knowledge of the plot of the film, which presumably allows for a superior situation model, thus suppressing MW. However, situational interest interacts with this relationship in that prior-knowledge alone is not enough to suppress MW when interest is low.

Author note This research was supported by the National Science Foundation (NSF) (DRL 1235958). Any opinions, findings and conclusions, or recommendations expressed in this paper are those of the authors and do not necessarily reflect the views of NSF. 


\section{Appendix}

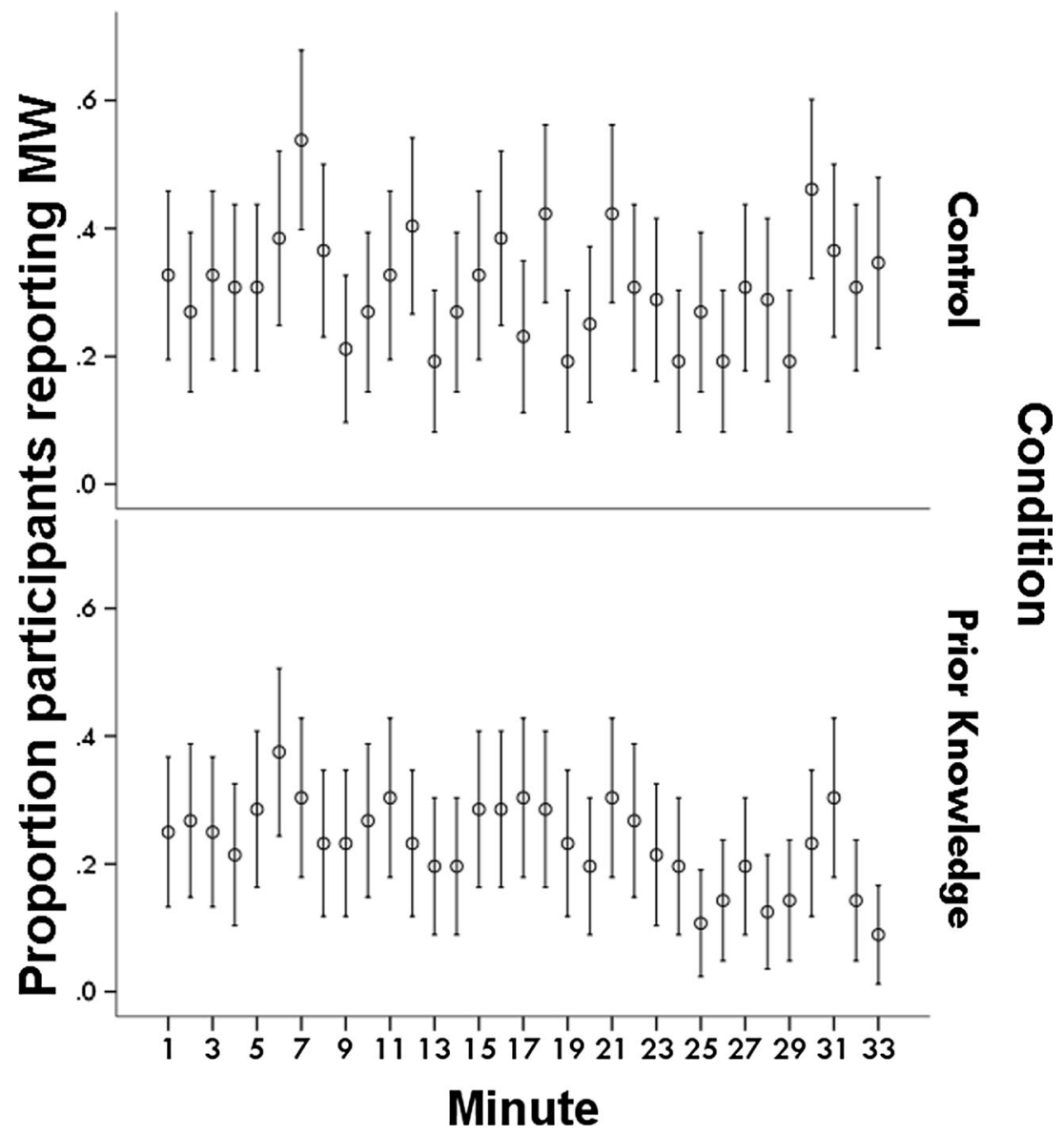

Fig. 3 Proportion of Participants Reporting MW in Each Minute of Film Viewing by Condition

\section{References}

Bates, D. M., \& Maechler, M. (2010). Ime4: Linear mixed-effects models using S4 classes. Retrieved from http://CRAN.R-project.org/ package $=$ lme 4

Bordwell, D. (2013). Narration in the fiction film. Routledge.

Faul, F., Erdfelder, E., Lang, A. G., \& Buchner, A. (2007). G*Power 3: A flexible statistical power analysis program for the social, behavioral, and biomedical sciences. Behavior Research Methods, 39(2), 175-191.

Giambra, L. M., \& Grodsky, A. (1989). Task-unrelated images and thoughts while reading. In J. E. Shorr, P. Robin, J. A. Connella, \& M. Wolpin (Eds.), Imagery: Current perspectives (pp. 27-32). New York, NY: Plenum Press

Graesser, A. C., Singer, M., \& Trabasso, T. (1994). Constructing inferences during narrative text comprehension. Psychological review, 101(3), 371.

Hidi, S. (2001). Interest, reading, and learning: Theoretical and practical considerations. Educational Psychology Review, 13(3), 191-209.

Hurlburt, R. T., Lech, B. C., \& Saltman, S. (1984). Random sampling of thought and mood. Cognitive Therapy and Research, 8(3), 263-275.

Klare, G. (1974). Assessing readability. Reading Research Quarterly, 10(1), 62-102.

Lamorisse, A. (1978). The Red Balloon. New York: Doubleday.
Linnenbrink-Garcia, L., Durik, A. M., Conley, A. M., Barron, K. E., Tauer, J. M., Karabenick, S. A., \& Harackiewicz, J. M. (2010). Measuring situational interest in academic domains. Educational and Psychological Measurement, 70(4), 647-671.

Magliano, J. P., Loschky, L. C., Clinton, J., \& Larson, A. M. (2013). Is reading the same as viewing? An exploration of the similarities and differences between processing text- and visually based narratives. In B. Miller, L. Cutting, \& P. McCardle (Eds.), Unraveling the Behavioral, Neurobiological, \& Genetic Components of Reading Comprehension (pp. 78-90). Baltimore, MD: Brookes Publishing Co.

Magliano, J. P., Miller, J., \& Zwaan, R. A. (2001). Indexing space and time in film understanding. Applied Cognitive Psychology, 15(5), 533-545.

McNamara, D. S., \& Magliano, J. (2009). Toward a comprehensive model of comprehension. Psychology of learning and motivation, 51, 297-384.

McVay, J. C., \& Kane, M. J. (2010). Does MW reflect executive function or executive failure? Comment on Smallwood and Schooler (2006) and Watkins (2008). Psychological Bulletin, 136, 188-197.

McVay, J. C., \& Kane, M. J. (2012). Why does working memory capacity predict variation in reading comprehension? On the influence of MW and executive attention. Journal of experimental psychology: general, 141(2), 302.

Pinheiro, J. C., \& Bates, D. M. (2000). Mixed-effects models in S and SPLUS. New York: Springer Verlag. 
Preacher, K. J., \& Hayes, A. F. (2004). SPSS and SAS procedures for estimating indirect effects in simple mediation models. Behavior Research Methods, Instruments, \& Computers, 36(4), 717-731.

Reise, S. P., \& Hurlburt, R. T. (1987). The relations between dimensions of thought reported in two thought-sampling studies. Imagination, Cognition and Personality, 7(4).

Risko, E. F., Buchanan, D., Medimorec, S., \& Kingstone, A. (2013). Everyday attention: mind wandering and computer use during lectures. Computers \& Education, 68, 275-283.

Schooler, J. W., Reichle, E. D., \& Halpern, D. V. (2004). Zoning out while reading: Evidence for dissociations between experience and metaconsciousness. Thinking and seeing: Visual metacognition in adults and children, 203-226.

Smallwood, J. (2011). Mind-wandering while reading: Attentional decoupling, mindless reading and the cascade model of inattention. Language and Linguistics Compass, 5(2), 63-77.

Smallwood, J., Fishman, D. J., \& Schooler, J. W. (2007). Counting the cost of an absent mind: MW as an underrecognized influence on educational performance. Psychonomic Bulletin \& Review, 14(2), 230-236.

Smallwood, J., Nind, L., \& O'Connor, R. C. (2009). When is your head at? An exploration of the factors associated with the temporal focus of the wandering mind. Consciousness and cognition, $18(1), 118-125$.

Smallwood, J., Obonsawin, M., \& Heim, D. (2003). Task unrelated thought: The role of distributed processing. Consciousness and cognition, 12(2), 169-189.

Smallwood, J., \& Schooler, J. W. (2006). The restless mind. Psychological bulletin, 132(6), 946.

Smallwood, J., \& Schooler, J. W. (2015). The science of Mind Wandering: Empirically navigating the stream of consciousness. Annual Review of Psychology, 66(1).

Smith, K. (1999). Bernie the Early Bloomer. Retrieved from http://www. bedtime.com/html/bernie_the_early_bloomer.htm

Stawarczyk, D., Majerus, S., Maj, M., Van der Linden, M., \& D'Argembeau, A. (2011). Mind-wandering: phenomenology and function as assessed with a novel experience sampling method. Acta Psychologica, 136(3), 370-381.

Zacks, J. M., Speer, N. K., Swallow, K. M., \& Maley, C. J. (2010). The brain's cutting-room floor: Segmentation of narrative cinema. Frontiers in human neuroscience, 4, 1-15.

Zwaan, R. A., \& Radvansky, G. A. (1998). Situation models in language comprehension and memory. Psychological Bulletin, 123(2), 162. 